Document downloaded from:

http://hdl.handle.net/10251/103152

This paper must be cited as:

Triana-Infante, CA.; Pastor Abellán, D.; Varón, M. (2017). Code Division Multiplexing Applied to FBG Sensing Networks: FBG Sensors Designed as Discrete Prolate Spheroidal Sequences (DPSS-FBG Sensors). Journal of Lightwave Technology. 35(14):2880-2886. doi:10.1109/JLT.2017.2705283

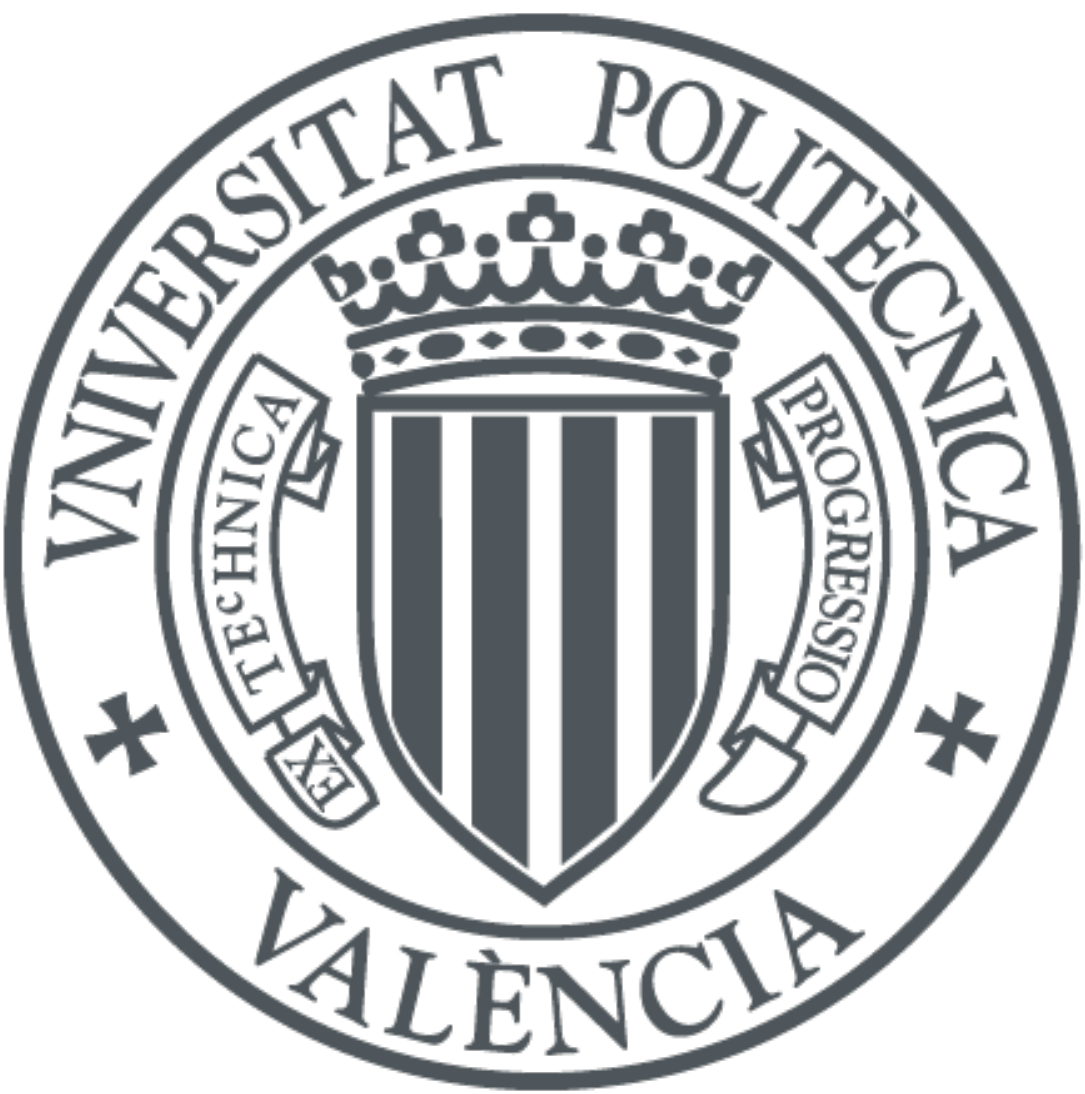

The final publication is available at

http://doi.org/10.1109/JLT.2017.2705283

Copyright Institute of Electrical and Electronics Engineers

Additional Information 


\title{
Code Division Multiplexing applied to FBG Sensing Networks: FBG Sensors Designed as Discrete Prolate Spheroidal Sequences (DPSS-FBG Sensors)
}

\author{
Andrés Triana, Daniel Pastor, Member, IEEE, and Margarita Varón, Member, IEEE
}

\begin{abstract}
We propose and demonstrate a sensing system with fiber Bragg grating (FBG) sensors designed as Discrete Prolate Spheroidal Sequences (DPSS). In this way, we can encode all of the sensing devices in the network making sure that each sensor is completely distinguishable from each other even under overlapping conditions. Since these devices are complex structures involving unique magnitude and phase response, the demodulation method should be able to recover the associated magnitude and phase response in order to identify each device in the sensing network. To do so, we use a Single Side Band (SSB) modulated optical source sweeping over the operational wavelength range of the sensors, the reflected signal from the sensors is sent to a Vector Network Analyzer (VNA) in which it is obtained the magnitude and phase ratio of the network in the microwave domain. Experimental demonstration has been carried out including the manufacturing of DPSS structures. Feasibility of the interrogation technique for devices involving magnitude and phase distinction has been validated, not only to identify encoded sensors in a measurement network but also to allow overlapping between them, which allow to increase the number of sensors allocated in the sensing network.
\end{abstract}

Index Terms-Optical fiber sensors, Sensor networks, Fiber Bragg gratings, FBG, Interrogation, Correlation, Overlap proof.

\section{INTRODUCTION}

$\mathbf{O}$ PTICAL fiber sensing technologies have attained great interest over the past years, this is due to the inherent advantages of the optical fiber such as, immunity to electromagnetic interference, low losses, among others [1], [2]. Fiber Bragg Gratings (FBGs) are a very mature technology used in optical sensing systems. Often, FBG sensing systems are interrogated in a simple broadband scheme based in Wavelength Division Multiplexing (WDM), in such systems the requirement for the sensors to not overlap sets the limit in the number of sensors that can be deployed in a single sensing system. Time Domain Multiplexing (TDM) has been widely used as an alternative to this problem [3] but, in general, it compromises the acquisition rate of the system and demands high speed switching circuits, recent approaches use weak FBGs to achieve large-scale sensing systems. Weak FBG sensors should be interrogated either with TDM [4] or frequency shifted interferometry [5] techniques, however, these

Andrés Triana and Daniel Pastor are with the Photonics Research Labs (PRL), Universitat Politècnica de València, Valencia 46022, Spain (e-mail: catrianai@unal.edu.co,dpastor@dcom.upv.es)

Andrés Triana and Margarita Varón are with the CMUN Research Group, Universidad Nacional de Colombia. 111321 Bogotá DC, Colombia

Manuscript received; approaches exhibit crosstalk limitations due to the shadowing effect between near-identical FBGs [6].

Nowadays, tailored FBG sensors can be manufactured in a routine way. In fact, manufacturing of 'complex' devices has been successfully achieved for example for encryption in Optical Code Division Multiple Access (OCDMA) communication systems [7]. In OCDMA, several approaches have been carried out, see [8], [9], recently, Discrete Prolate Spheroidal Sequences (DPSS) have been also proposed [10] and experimentally validated [11] showing a high encryption potential and very suitable features for channeling in WDM systems due to their fixed optical bandwidth.

The accurate and repeatable manufacturing process of SuperStructured Fiber Bragg Gratings (SSFBG) suggests the idea of manufacturing more elaborated structures to act as sensors in an optical sensing network, therefore, combining the sensing properties of standard FBG sensors with the advantages of encoding in optical systems would definitely be a major progress leading to enhance the performance of sensing systems. Note that since such structures will be manufactured as a compound of individual FBGs, they will preserve the sensing properties of any standard FBG sensor. Indeed, we have demonstrated in previous works [12]-[14] that providing the sensors with a distinctive amplitude shape makes it possible to achieve orthogonality between them, in such a way that each sensor in the network can be unequivocally distinguished even in overlapping scenarios. It is worth noting that other sensing systems have included CDMA concepts before [15], nevertheless the encoding of this kind of systems occurs in the source, not in the shape of the sensors, so they are more likely to be classified as a variation of a TDM. Also, the problem of identifying two or more overlapped FBG sensors has been addressed previously, for instance [16] proposed the use of bio-inspired software techniques to find each sensor's central wavelength in overlapping scenarios, but these techniques are non-deterministic and time consuming.

In this paper, we propose the use of code division multiplexing in order to resolve optical FBG sensors. The main difference with our previous work relies in the fact that here we use magnitude and phase patterns to encode the FBG sensors while in our previous approaches [12]-[14] we were shaping the sensors only in amplitude. Still, the fundamental idea is to add another multiplexing dimension, compatible with traditional WDM schemes, increasing the number of sensors that can be deployed in the system. Therefore, Discrete Prolate Spheroidal Sequences (DPSS) are manufactured as super structured FBGs 


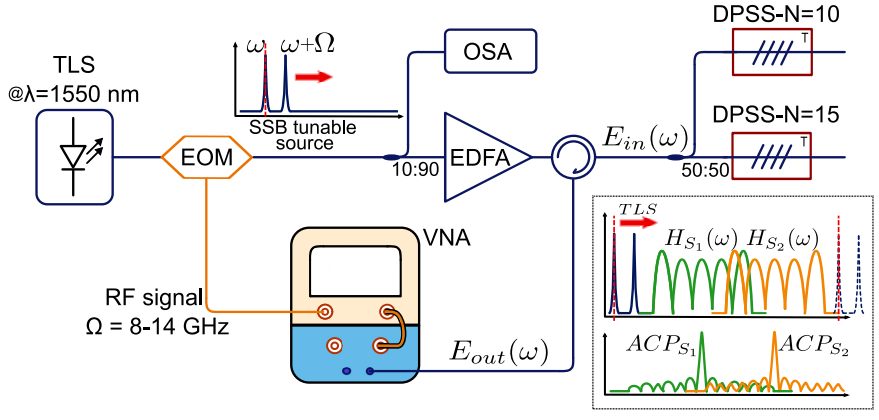

Figure 1. A parallel-schematic sensing setup is implemented to measure the central wavelength for each one of the DPSS-FBG sensors. A tunable dualband source $E_{i n}(\omega)$ is swept over the working spectrum; the reflected signals $E_{\text {out }}(\omega)$ are retrieved at the VNA to determine their frequency response at a fixed $\Omega$. Finally, the cross-correlation product (see Inset.) is performed to obtain the central wavelength $A C P$ of each sensor in the network. TLS, tunable laser source; SSB, single side band modulation; EOM, electrooptic modulator; OSA, optical spectrum analyzer; EDFA, erbium-doped fiber amplifier; DPSS, discrete prolate spheroidal sequence sensor; VNA, Vector Network Analyzer; $H_{S_{k}}(\omega)$, frequency response of each sensor, $A C P_{S_{k}}$ Auto-correlation peak for each sensor.

and used as sensor devices with a very definite (magnitude and phase) pattern. This fact not only allows the sensors to be recognizable under overlapping scenarios but also adds robustness to the sensing system in the sense that the measured information has a very definite shape regarding interference or distortion in the spectrum.

The proposed interrogation scheme (Fig. 1), which is demonstrated in this paper for two sensing devices, makes use of a tunable laser source Single Side Band (SSB) modulated to a fixed microwave frequency, sweeping over the operational wavelength range of the sensors. The reflected signal from the set of DPSS sensors (which contains both, the carrier and the side band) beats at the photo detector on the Vector Network Analyzer (VNA) providing the magnitude and phase ratio of the sensing elements in the network evaluated at the fixed modulating microwave signal. When the complete profile of the sensing network is obtained, a correlation product between the theoretical and the measured response of the sensors is performed. From this, the auto-correlation peak (ACP) is obtained, pointing out the central position for each DPSS sensor in the network, with the additional advantage that two or more sensors can be overlapped in the same operational wavelength range.

Although other systems have employed a dual band in the past [17], this is the first time, that a dual-band source is employed in an interrogation system to retrieve the complex (magnitude and optical phase) wavelength response of sensing devices. This interrogation method enables the possibility to identify complex encoded sensors designed with a unique spectral shape like the DPSS-FBG sensors. Thus, the number of sensors arranged in a sensing network is increased since the orthogonality between them permits two or more sensors to operate in the same spectral region, being their central wavelengths completely identifiable from each other. Note that this concept of code multiplexing is compatible with WDM and other multiplexing schemes frequently used in FBG systems. The rest of the paper is organized as follows, in section II it is

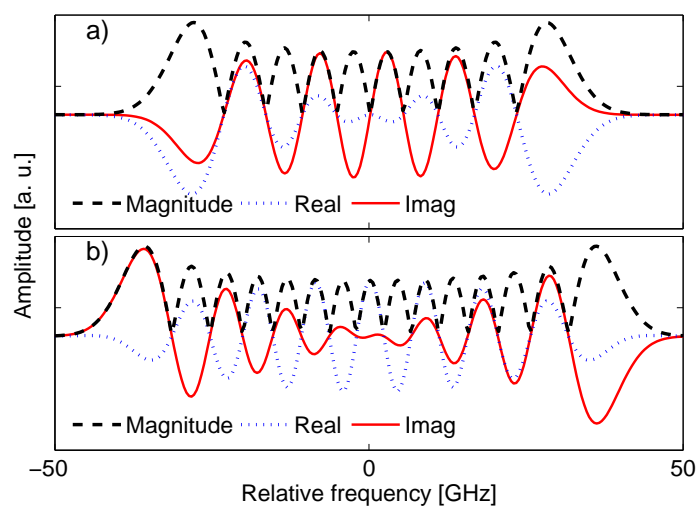

Figure 2. Theoretical frequency response of the DPS sequences; real part: blue (dotted line), imaginary part: red (continuous line), magnitude: black (dashed line). a): DPS Sequence with $k=10$. b): DPS Sequence with $k=15$.

described the operation principle of the interrogation system, first, the DPS sequences are described and their manufacturing process as SS-FBG devices is detailed. Next, the demodulation principle is described and some considerations are discussed, then, the correlation process required to obtain the central wavelength of each sensor from the total frequency response of the system is explained. In section III a experimental proof-ofthe-concept system with two DPSS-FBG sensors is validated, obtaining complete identification of the central wavelength for each sensor in the network even when the sensors are overlapping. Finally, Some conclusions are drawn in section IV.

\section{PRINCIPLE}

The schematic of the proposed interrogation system is presented in Fig. 1. The main idea behind the dual-band tunable source in the interrogation system is to identify elaborated sensor structures in a network by comparing the magnitude and phase pattern reflected by each sensor in the system. A very good candidate for these elaborated structures are the discrete prolate spheroidal sequences (DPSS), explained below.

\section{A. DPSS structures and manufacturing of DPSS-FBG sensors}

As mentioned before, the DPS sequences may be seen as a set of optical devices, which have two interesting properties for optical communication systems: they are mutually orthogonal regardless of the sequence order and, they are frequency band limited to a delimited range of frequencies; given this 'orthogonal-delimited in spectrum' nature, it is possible to think about DPSS-shaped FBG sensors that can be constrained into a fixed spectrum range, more importantly: whose central wavelength can be identified under overlapping conditions.

To compute a set of $N$ DPSS codes, the eigen-vectors of a symmetric tridiagonal matrix $T(N, W)$ must be found [18]. After defining $N$, the number of orthogonal codes and $W$, the discrete frequency covering range. The entries of the tridiagonal matrix are given by (1).

For our experimental validation we have selected $N=128$ and $W=0.5$. Which means that we have 128 orthogonal codes of 128 chip length. Fig. 2 shows the spectral response 


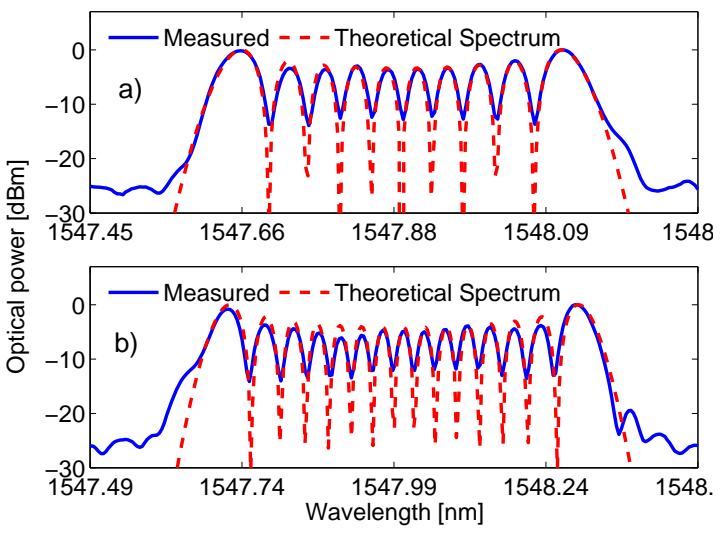

Figure 3. Measured spectrum for FBG-manufactured DPS sequences with a): $k=10$ and $\mathbf{b}$ ): $k=15$. Manufactured spectrum, plotted in blue (continuous line), is compared with the theoretical expected spectrum in red (dashed line).

of the sequences number $k=10$, and 15 , it is represented the real part in blue (dotted line), the imaginary part in red (continuous line) and the magnitude in black (dashed line). The number of the sequences in the set, from $k=1$ to $N$, corresponds with the degree concentration of the energy in the spectral and temporary domains and with the number of alternating lobes of the structure. Besides, Fig. 2 displays the behavior differences (real and imaginary parts) between even and odd DPS sequences.

$$
T(i, j)= \begin{cases}\frac{1}{2} i(N-1), & j=i-1 \\ \left(\frac{N-1}{2}-i\right)^{2} \cos (2 \pi W), & j=i \\ \frac{1}{2}(i+1)(N-1-i), & j=i+1 \\ 0, & \text { otherwise } \\ , \quad i, j=0,1, \ldots, N-1\end{cases}
$$

In the DPSS-FBG sensor design we employed the Discrete Layer Peeling (DLP) synthesis method, targeting on the ideal spectral response represented in Fig. 2. Although the length of the sequences is $N=128$ this information was linearly interpolated after de DLP process to obtain a complex (magnitude and phase) index perturbation profile $(q(z)$ ), sampled each $\sim 85.6 \mu \mathrm{m}$. Each point is manufactured through UltraViolet (UV) laser beam exposition after a Phase Mask (PM) to obtain the Bragg pattern. UV beam was focused before the PM up to $\sim 40 \mu \mathrm{m}$. Magnitude and phase control of each sample of $q(z)$ was achieved by a double UV exposition of $200 \mathrm{~ms}$ ( $50 \mathrm{~mW} \mathrm{UV}$ beam) over the same z-position, only changing the relative phase of the Bragg period between them by the proper displacement of the Bragg pattern. In this way, the averaged UV flux is constant and the averaged value of the refractive index remains unaltered [19], [20]. After manufacturing, DPSS sensors' shape was assessed by a direct power spectral measurement employing an amplified spontaneous emission (ASE) source and an optical spectrum analyzer (OSA) with $10 \mathrm{pm}$ resolution. Fig. 3 compares the manufactured and the theoretical spectra.

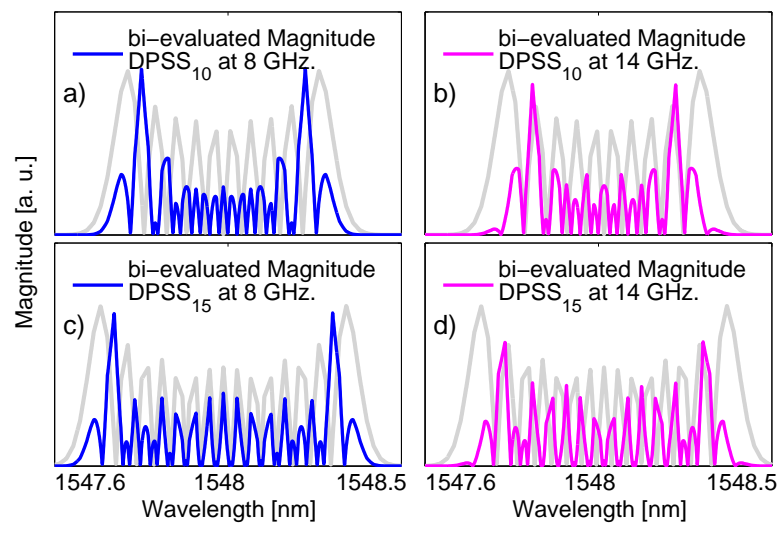

Figure 4. Bi-evaluated frequency response for the DPSS-FBG sensors studied in this work. These are the signals employed in the cross-correlation product to obtain DPSS-FBG sensors' central wavelength. a), c): Magnitude of the bi-evaluated frequency response at $\Omega=8 \mathrm{GHz}$. for codewords 10 and 15 respectively b), d): Magnitude of the bi-evaluated frequency response at $\Omega=14 \mathrm{GHz}$. for codewords 10 and 15 respectively (Watermark shows the magnitude of the actual DPSS-FBG sensors for clarity).

\section{B. Demodulation}

For the demodulation of the DPSS-shaped sensors it is used a dual-band source which is obtained, as depicted in the schematic setup (Fig. 1), from the SSB modulation of a TLS. At the same time as the TLS is swept over the wavelength operational range of the sensors, the Vectorial Network Analyzer is employed to measure the electro-optical frequency response of the complete system $H(\Omega, \lambda)$ or $H(\Omega, \omega)$ for a fixed microwave angular frequency $\Omega$ (i.e. VNA measurement of the scattering parameter $S_{21}$, considering the sensing network as an optical transmission system.).

Initially, we can model the SSB interrogating signal $\left(E_{\text {in }}(\omega)\right.$ in Fig. 1) as (2).

$$
E_{\text {in }}(\omega)=a_{1} \delta(\omega)+a_{2} \delta(\omega+\Omega)
$$

In (2) $\omega$ is the optical angular frequency $\omega=2 \pi c / \lambda$, being $c$, the speed of light in vacuum, $\lambda$, the source wavelength along the sweep, $a_{1}$ and $a_{2}$ the arbitrary amplitudes of the two bands and $\delta$ denotes the Dirac's delta function. After propagation and reflection on the sensor network we can write the electrical field acceding to the VNA photodetector $\left(E_{\text {out }}(\omega)\right.$ in Fig. 1) as (3).

$$
E_{\text {out }}(\omega)=a_{1} H_{\text {net }}^{O}(\omega) \delta(\omega)+a_{2} H_{\text {net }}^{O}(\omega+\Omega) \delta(\omega+\Omega)
$$

In (3), $H_{n e t}^{O}$ denotes the optical complex spectral response of the network including the propagation round trip paths. Finally, taking inverse Fourier transformation to work in time domain the detected photo current will be proportional to (4).

$$
\begin{aligned}
i(t) \propto & \left|H_{n e t}^{O}(\omega)\right|\left|H_{n e t}^{O}(\omega+\Omega)\right| \\
& \quad \cos \left(\Omega t+\left[\angle H_{n e t}^{O}(\omega)-\angle H_{n e t}^{O}(\omega+\Omega)\right]\right)
\end{aligned}
$$

Notice that in (4) we have not included the baseband terms $\omega=0$. The VNA standard processing filters out the microwave signal located at the $\Omega$ from the detected photocurrent, then it is compared (in magnitude and microwave phase) with the 


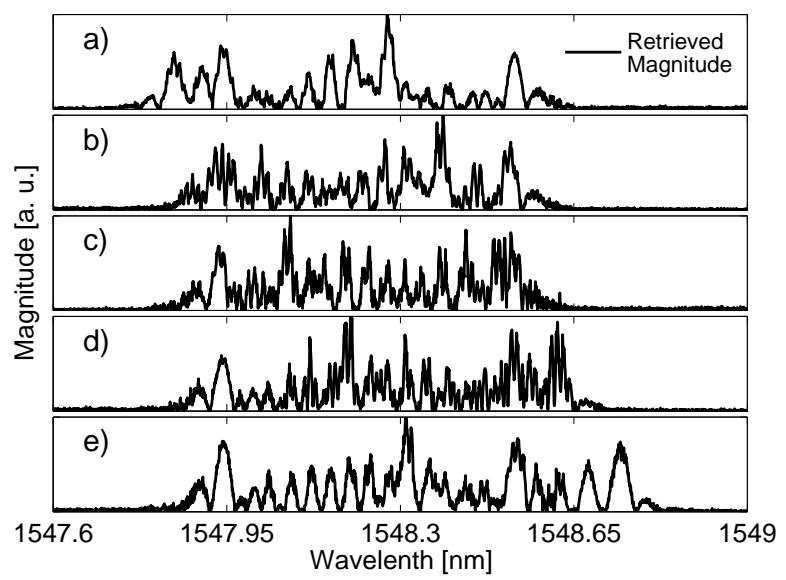

Figure 5. Experimental measurement of the frequency response of a sensing network composed of two DPSS-FBG sensors at $8 \mathrm{GHz}$. Labels a) to d) show four different captures of the system as one of the sensors is wavelength shifted while the second one is maintained at a fixed wavelength

modulating signal employed in the SSB generation. This process provides the complete electro-optical frequency response (at the fixed RF value of $\Omega$ ) as (5).

$$
\begin{gathered}
|H(\Omega, \omega)| \propto\left|H_{n e t}^{O}(\omega)\right|\left|H_{n e t}^{O}(\omega+\Omega)\right| \\
\angle H(\Omega, \omega)=\angle H_{n e t}^{O}(\omega)-\angle H_{n e t}^{O}(\omega+\Omega)
\end{gathered}
$$

From (5), it is worth to mention that magnitude and optical phase from the optical network $\left(H_{n e t}^{O}(\omega)\right)$ are obtained as a double evaluation (at $\omega$ and $\omega+\Omega$ ) over the entire spectrum, being critical the proper sensor design and the microwave angular frequency $(\Omega)$ selected in the demodulation system. Considering that the frequency response $H(\Omega, \omega)$ of the system is obtained by a dual-band source, the correlation product $(C P)$ (which is a scalar product between the total frequency response of the system and the individual expected responses of all the sensors in the network intended to compute their central wavelengths) should be performed for each theoretical DPSS-FBG sensor in the network, also evaluated at the two frequencies of interest $(\omega, \omega+\Omega)$ (i.e. each sensor bi-evaluated theoretical response). Fig. 4 shows the magnitude of the $b i$ evaluated theoretical response for the DPSS-FBG sensors employed in the experimental demonstration DPSS $_{10}$ and DPSS $_{15}$. Fig. 4 a), c) shows the bi-evaluated theoretical response evaluated at $\Omega=8 \mathrm{GHz}$ while Fig. $4 \mathrm{~b}$ ), d) depicts the equivalent evaluated at $\Omega=14 \mathrm{GHz}$. The differences between the bi-evaluated responses and the actual spectral shape of the DPSS-FBG sensors (watermark in Fig. 4) are noticeable. Moreover, the bi-evaluated response for a determinate sensor changes for different values of the modulation frequency $\Omega$.

To obtain the central wavelength of each sensor $\left(\lambda_{B_{k}}\right)$, it is performed the correlation product $C P_{k}$ between the total frequency response of the sensing network $H(\Omega, \omega)$, and the bi-evaluated frequency response of the $k$-th DPSS-FBG sensor in the network, this can be written as (6).

$$
C P_{k}\left(\omega^{\prime}\right)=\int H(\Omega, \omega) \cdot H_{S_{k}}\left(\omega^{\prime}\right) d \omega
$$

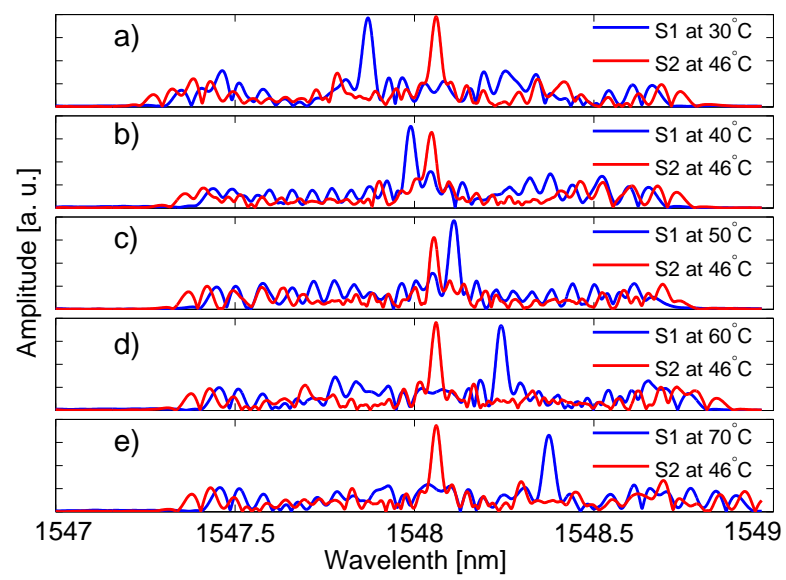

Figure 6. The correlation product is computed for the captured $8 \mathrm{GHz}$. frequency response of the sensing network. The obtained ACP determines the central wavelength for each sensor deployed in the network.

The result of the correlation product $C P_{k}$ is composed of the Auto-Correlation Peak (ACP), indicating the central wavelength $\lambda_{B_{k}}$ of the matching DPSS-FBG sensor in the spectrum, and the cross-correlation (XC) corresponding to the remaining spectral signals in the sensing network (XC signal is constrained to a low level due to the orthogonality of the DPSS codes). The correlation product is computed over the entire measured spectrum for each one of the $k$ DPSS-FBG sensors deployed in the network. A key advantage is that this is a simple scalar product between two signals, so it does not add either time delay or complexity to the interrogation process.

The correlation process plays an important role apart from detecting each sensor's central wavelength. It can be seen also as a post-processing filtering stage that bypasses unwanted components from the measured signal. For instance, beat noise is produced between overlapping sensors, causing an additional ripple to the measured signal. The ripple's frequency depends on the distance between two sensors that form a cavity when they overlap. However, when the correlation product is performed, ripple signals are disregarded because each sensor's expected response has a very specific shape. Beat noise is an important issue in optical CDMA communications systems, where transmitted information needs to be detected at bit-rate speed. Contrarily, in sensing systems the beat noise effect can be neglected by additional filtering stages given that post-processing time is not critical.

\section{EXPERIMENT}

A proof-of-concept experiment with two orthogonal DPSSFBG sensors was performed. This experiment follows the setup depicted in Fig. 1, where the TLS is a narrow-linewidth tunable laser (Yenista TUNICS T100R). The laser is tunable in the $1520-1620 \mathrm{~nm}$. In our experiment (see Fig. 1), TLS is swept at $1 \mathrm{~nm} / \mathrm{s}$ and modulated by a dual-drive Mach-Zender modulator at 8 and $14 \mathrm{GHz}$ to obtain the dualband interrogation signal. Then, dual-band signal $E_{i n}(\omega)$ is amplified and guided to the sensing devices. The light goes through the sensor devices, whose spectrum is shown in Fig. 3 


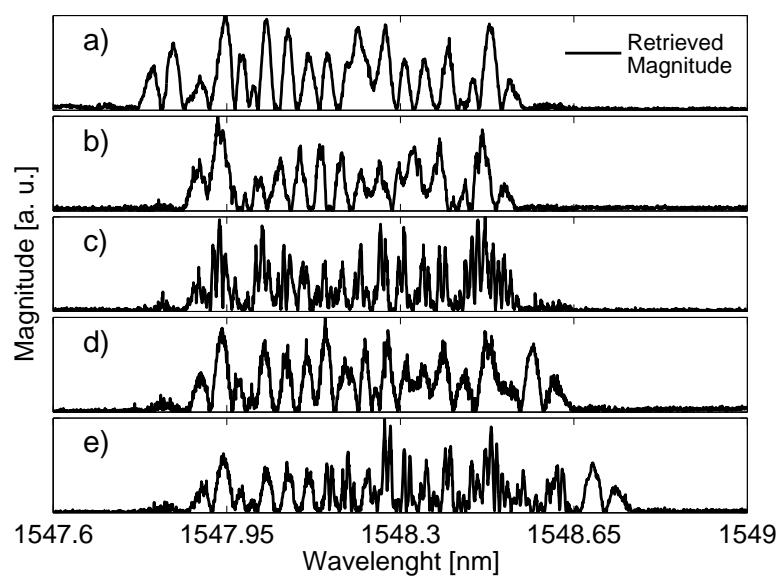

Figure 7. Experimental measurement of the frequency response of a sensing network composed of two DPSS-FBG sensors at $14 \mathrm{GHz}$. Labels a) to d) show four different captures of the system as one of the sensors is wavelength shifted while the second one is maintained at a fixed wavelength.

and is reflected according with the shape of each sensor. Then, the reflected signal $E_{\text {out }}(\omega)$ is received by the VNA which obtains the frequency response of the sensing network (magnitude and phase). Two passive splitters are used in the setup, the first one is a 10:90 splitter to monitor the dualwavelength signal at the OSA and the second one is a 50:50 splitter used for parallel configuration of the two DPSS-FBG sensors. The two sensors are temperature controlled, one of them is maintained at a fixed temperature while the other one is shifted in wavelength to prove the demodulation process under overlapping conditions. The instant frequency response of the system is captured by the VNA at each step of the experiment and the readout is transferred to a computer to perform the correlation-based demodulation, obtaining as a result the central position for each sensor in the network.

As mentioned in section II-B, the modulation frequency $(\Omega)$ used to generate the dual-wavelength interrogation signal enhances the correlation process at some specific frequencies. To verify this, we have measured the frequency response of the sensing network at two frequencies: $\Omega=8$ and $14 \mathrm{GHz}$. Fig. 5 shows the magnitude of the frequency response measured by the VNA at $\Omega=8 \mathrm{GHz}$. Fig. 5 a)-d) shows the instantaneous readout of the experiment as one sensor is shifted in five temperature steps while the second sensor remain at a fixed spectral position. As the DPSS-FBG sensors are intended to be overlapping in the spectrum, the captured frequency response of the system contains the aggregate of all the individual contributions in the network. The demodulation principle for this experiment consists on the correlation between the measured frequency response of the entire network and the individual frequency response of each sensor deployed in the network (6). Fig. 6 shows the correlation product obtained for each readout depicted in Fig. 5. The ACP is capable of demodulating two sensors, even when they are overlapping in the same spectral region, due to the simple fact that each sensor in the network has a unique complex spectral shape, i.e. we are using a code division multiplexing (CDM) scheme with DPSS

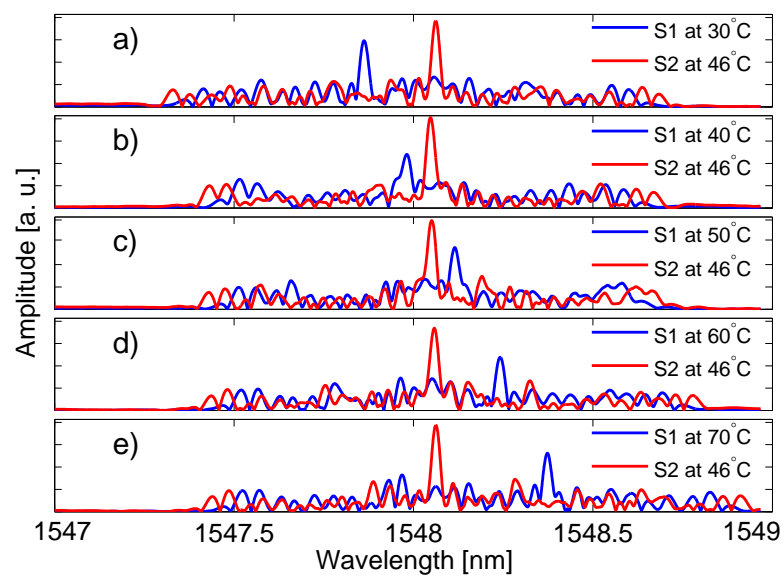

Figure 8. The correlation product is computed for the captured $14 \mathrm{GHz}$. frequency response of the sensing network. The obtained ACP determines the central wavelength for each sensor deployed in the network.

codes to shape the FBG sensors. Note that this CDM concept is compatible with WDM multiplexing schemes so the scenario can be extended to CDM-WDM multiplexing networks where each DPSS-FBG sensor would have a unique spectral shape regarding its neighboring sensors in the same WDM channel. In this way, the maximum number of sensors allocated in the sensing network depends on the number of DPSS-FBG sensors manufactured for each WDM channel, the simplest scheme is the one used in this proof-of-concept, which allows two sensors per channel, consequently doubling the number of sensors deployed in the network.

The same experiment has been carried out setting the modulation frequency to $\Omega=14 \mathrm{GHz}$. The main idea behind this is to verify that the ACP/XC ratio is different for each DPSSFBG sensor when varying $\Omega$. Fig. 7 a)-d) shows the frequency response of the sensing network during the experiment at $\Omega=14 \mathrm{GHz}$. Whereas Fig. 8 depicts the resultant cross correlation product for each readout in the experiment. It is clear from the comparison between the ACP signals obtained in Figs. 6 and 8 that the ratio $\mathrm{ACP} / \mathrm{XC}$ has a dependence relationship with the modulation frequency used to interrogate the system. This, in contrast to being a disadvantage, is positive because it enables the possibility of interrogating the system at different modulation frequencies in order to enhance the response of a given sensor for each sweep of the identification procedure.

At this point, it is evident from the experimental measurement that the correlation product of sensor $S 1$, for example, has a better dynamic range when the frequency response of the system is measured at $8 \mathrm{GHz}$. To elucidate how the dynamic range of each sensor is affected by the modulation frequency of the system $(\Omega)$, a theoretical simulation was performed. In Fig. 11, the dynamic range is calculated for sensors $S 1$ and $S 2$ while the modulation frequency is swept from 0 to $20 \mathrm{GHz}$. Fig. 11 a) represents the worst-case-scenario in which the two sensors $S 1$ and $S 2$ are in the exact same spectral position, in this case $\Omega=8 \mathrm{GHz}$ and $\Omega=14 \mathrm{GHz}$ are the frequencies where the dynamic range of both sensors 


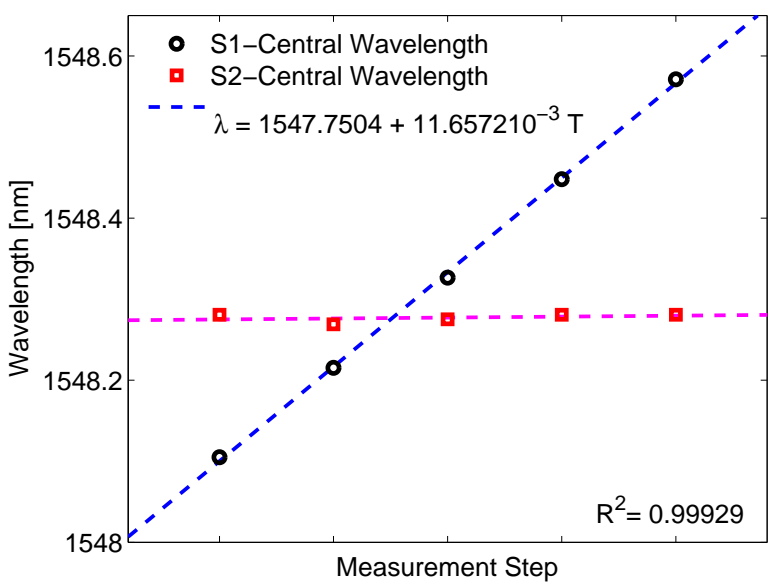

Figure 9. $8 \mathrm{GHz}$. Measured wavelength of two DPSS-FBG sensors in an overlapping scenario. One of the sensors remains steady while the second one is temperature shifted in the same operational wavelength region.

takes similar values. In this worst-case-scenario the calculated dynamic range displays values ranging between $44 \%$ and $60 \%$ (this means that the $\mathrm{XC}$ signal rise to take values between the 44 and $60 \%$ of the ACP signal at the frequencies of interest). On the other hand, Fig. 11 b) shows a regular scenario where the two sensors are barely overlapping. In this case, the dynamic range for sensors $S 1$ and $S 2$ ranges from $26 \%$ to $37 \%$. Note that even in the worst-case-scenario the detection capacity of the auto-correlation peak is feasible.

To further investigate the performance of the proposed sensors the center wavelength shift measured at the experiment is plotted for every measurement step in which one of the sensors is shifted in temperature. Fig. 9 shows the measurement performed at $\Omega=8 \mathrm{GHz}$, the overlap-proof behavior of the sensors is appreciated, circles represent the computed central wavelength for sensor $S_{1}$ showing linear relationship with the temperature and, squares represent the central wavelength of the steady sensor $S 2$. The dashed line shows the lineal fit to the data, obtaining a slope of $11.657 \mathrm{pm}$ with a very good coefficient of determination $R^{2}=0.9992$. The same analysis was made for the measurements taken at $\Omega=14 \mathrm{GHz}$ (Fig. 10). Results were very consistent, in this case the slope obtained from the linear fit is $11.855 \mathrm{pm}$ with a coefficient of determination $R^{2}=0.9997$. This confirms the fact that superstructured FBG like the DPSS-FBG sensors proposed here retain the same sensitivity of conventional FBG sensors. In other words, all reflection sub-bands in the device are affected in the same proportion by environmental changes surrounding the optical fiber.

\section{CONCLUSION}

We have proposed the use of DPS sequences in the design of FBG sensors taking advantage of their orthogonal nature. Thus, we add code division multiplexing to the sensing network increasing consequently the maximum number of sensors regarding an analogous WDM system. DPS sequences are a very robust set of codes optically orthogonal between them. This feature, when applied to the design of optical

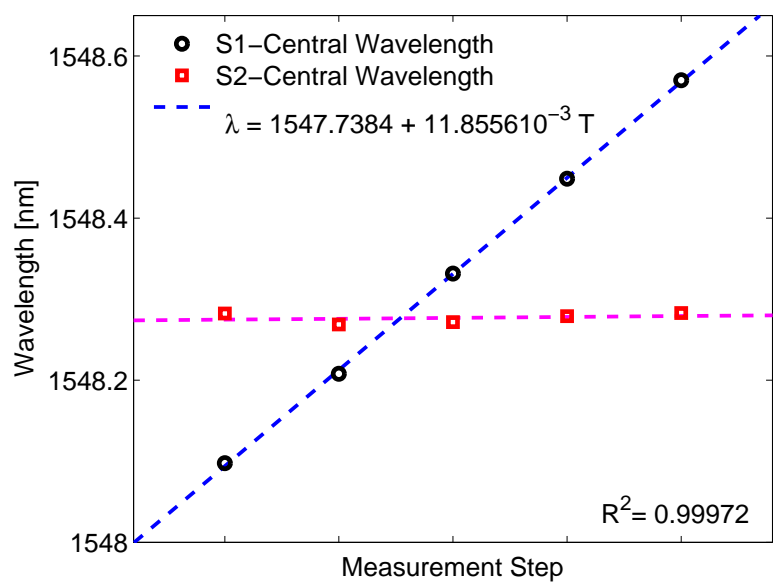

Figure 10. $14 \mathrm{GHz}$. Measured wavelength of two DPSS-FBG sensors in an overlapping scenario. One of the sensors remains steady while the second one is temperature shifted in the same operational wavelength region.

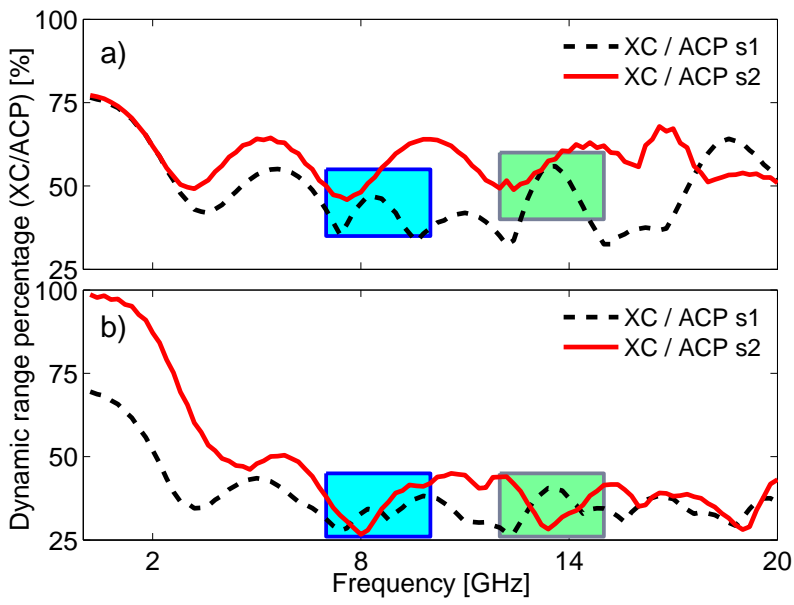

Figure 11. Dynamic range calculated for sensors $S 1$ and $S 2$ at different values of the modulation microwave frequency $\Omega$. Highlighted regions show the selected $\Omega$ values utilized in the experimental validation. a) shows the $\mathrm{XC} / \mathrm{ACP}$ ratio for both sensors at the worst-case-scenario: identification of two sensors at the same spectral location. b) shows the calculated XC/ACP ratio for the two sensors when their spectral positions are barely overlapping.

FBG sensors, allows total identification in the network of each sensor device even in scenarios of overlapping between two sensors. Interestingly, DPSS have also the property of being restricted to a certain design bandwidth. This is a key difference with other sets of orthogonal codes that require longer codewords to increase their cardinality. In our work, we designed DPS sequences within a bandwidth of $0.8 \mathrm{~nm}$ thus we can guarantee CDM-WDM compatibility since all the DPSS-FBG sensors will have approximately the same bandwidth. Given that the DPSS structures were manufactured as a complex index profile FBG, all the structure follows the behavior of conventional FBGs, i.e. DPSS-FBG sensors have the same resolution due to environmental variables affecting the optical fiber. Accuracy, on the other hand, relies mainly on the source specifications, in our case the laser has a minimal incremental wavelength resolution of $1 \mathrm{pm}$. There is, nevertheless, a 'crosstalk' effect between the ACP and 
the XC lobes in the correlation algorithm. This penalty was experimentally measured in [13] as $\pm 3 \mathrm{pm}$.

Experimental validation of the proposed sensors was achieved. In the experiments, frequency response of the sensing network was measured at a fixed microwave angular frequency $\Omega=8 \mathrm{GHz}$ and $\Omega=14 \mathrm{GHz}$. This was done to show how the $\Omega$ enhances the dynamic range in the measurement of certain sensors' profiles. In fact, it was evidenced that the ratio $\mathrm{ACP} / \mathrm{XC}$ depends on the modulation frequency used $(\Omega)$. Besides, a theoretical simulation was performed to show how the dynamic range of each sensor (relationship between the ACP and the XC signals) is affected by the modulation frequency at two very specific overlapping conditions: the worst-case-scenario when the two sensors are at the exact same position in the spectrum and a regular scenario when the two sensors are aside from each other. It was shown that the auto correlation peak (ACP) indicating the central wavelength $\lambda_{B_{k}}$ of each sensor can be always identified. The simulation showed that the $\mathrm{XC}$ is constrained to a maximum value that reaches the $60 \%$ of the ACP when two overlapping sensors are exactly at the same spectral position.

To assess the improvement of a sensing network designed with this CDM-WDM approach, we have demonstrated in this proof-of-the-concept paper that using 2 DPSS-FBG sensor devices per channel, it is possible to double the number of sensors allocated in the network when compared with an equivalent WDM sensing system. Although the laboratory equipment used in this experiment could be expensive, the cost could be reduced by replacing the VNA for a dedicated hardware implementation operating at a single frequency; even more, taking advantage of the upcoming development in integrated photonics, all of the components utilized in the system (i.e. lasers, modulator, detector) could be implemented in a single photonic chip [21], downscaling consequently overall costs.

\section{ACKNOWLEDGMENT}

This work was supported by the Spanish MCINN through projects TEC2016-80385-P, TEC2013-42332-P and TEC 2009-12169; and by the Colombian Science, Technology and Innovation department (Colciencias) through $\mathrm{PhD}$ call 567.

\section{REFERENCES}

[1] B.Culshaw and A.Kersey, "Fiber-optic sensing: A historical perspective," Journal of Lightwave Technology, vol. 26, no. 9, May 2008.

[2] A.Kersey et al., "Fiber grating sensors," Lightwave Technology, Journal of, vol. 15, no. 8, pp. 1442-1463, Aug 1997.

[3] M.Njegovec and D.Donlagic, "Interrogation of fbgs and fbgs arrays using standard telecom dfb diode," Journal of Lightwave Technology, vol. 34, no. 22, pp. 5340-5348, Nov 2016.

[4] J.Rohollahnejad et al., "Tdm interrogation of intensity-modulated usfbgs network based on multichannel lasers," Opt. Express, vol. 25, no. 2, pp. 670-680, Jan 2017

[5] Y.Ou et al., "Large-capacity multiplexing of near-identical weak fiber bragg gratings using frequency-shifted interferometry," Opt. Express, vol. 23, no. 24, pp. 31 484-31495, Nov 2015.

[6] H.Guo et al., "Crosstalk and ghost gratings in a large-scale weak fiber bragg grating array," Journal of Lightwave Technology, vol. 35, no. 10, pp. 2032-2036, May 2017

[7] S.Ayotte et al., "Experimental verification and capacity prediction of FEOCDMA using superimposed FBG," Lightwave Technology, Journal of, vol. 23, no. 2, pp. 724-731, Feb 2005
[8] X.Wang et al., "Ocdma over wdm transmission," in 2007 9th ICTON Conference, vol. 1, July 2007, pp. 110-113.

[9] C. C.Yang et al., "Performance analyses on hybrid mqc/m-sequence coding over frequency/spatial optical cdma system," IEEE Transactions on Communications, vol. 55, no. 1, pp. 40-43, Jan 2007.

[10] I. B.Djordjevic et al., "Design of dpss based fiber bragg gratings and their application in all-optical encryption, ocdma, optical steganography, and orthogonal-division multiplexing," Opt. Express, vol. 22, no. 9, pp. 10 882-10 897, May 2014.

[11] D.Pastor et al., "Coherent optical en/decoding employing discrete prolate spheroidal sequences based super structured fbgs," in Optical Cоттиnication (ECOC), 2015 European Conference on, 2015.

[12] A.Triana et al., "Overlap-proof fiber bragg grating sensing system using spectral encoding," IEEE Photonics Technology Letters, vol. 28, no. 7 pp. 744-747, April 2016.

[13] _ "Enhancing the multiplexing capabilities of sensing networks using spectrally encoded fiber bragg grating sensors," Journal of Lightwave Technology, vol. PP, no. 99, pp. 1-1, 2016.

[14] C. A.Triana et al., "Optical code division multiplexing in the design of encoded fiber bragg grating sensors," Optica pura y aplicada, vol. 49 , no. 1, pp. 17-28, Feb 2016, iSSN-e 2171-8814.

[15] A. D.Kersey et al., "Low-crosstalk code-division multiplexed interferometric array," Electronics Letters, vol. 28, no. 4, pp. 351-352, Feb 1992.

[16] H.Jiang et al., "Wavelength detection in spectrally overlapped fbg sensor network using extreme learning machine," Photonics Technology Letters, IEEE, vol. 26, no. 20, pp. 2031-2034, Oct 2014.

[17] A. M. R.Pinto et al., "Interrogation of a suspended-core fabry-perot temperature sensor through a dual wavelength raman fiber laser," Journal of Lightwave Technology, vol. 28, no. 21, pp. 3149-3155, Nov 2010.

[18] D.Slepian, "Prolate spheroidal wave functions, fourier analysis, and uncertainty - v: the discrete case," Bell System Technical Journal, The, vol. 57, no. 5, pp. 1371-1430, May 1978.

[19] R.Baños et al., "Rectangular Global Envelope Super Structured FBGs for Multiband Coherent OCDMA," Photonics Technology Letters, IEEE, vol. 25, no. 5, pp. 512-514, March 2013.

[20] _ "Chromatic dispersion compensation and coherent direct-sequence ocdma operation on a single super structured fbg," Opt. Express, vol. 20, no. 13, pp. 13966-13 976, Jun 2012

[21] J. S.Fandiño et al., "A monolithic integrated photonic microwave filter," Nat Photon, vol. 11, no. 2, pp. 124-129, Feb 2017.

Andrés Triana was born in Bogotá, Colombia, in 1988. He received the Bachelor's degree in electronics engineering and the M.Eng. degree in industrial automation, both from the Universidad Nacional de Colombia, Bogotá, in 2011 and 2013, respectively. He is currently working towards the Ph.D. degree in communications engineering at the Photonics Research Labs. (PRL) from Universitat Politècnica de València and Universidad Nacional de Colombia.

Daniel Pastor was born in Elda, Spain, on November 5, 1969. He received the Ingeniero de Telecomunicación degree and the Doctor Ingeniero de Telecomunicación (Ph.D.) degree from the Universitat Politècnica de València (UPV), Valencia, Spain, in 1993 and 1996, respectively. He joined the Departamento de Comunicaciones, UPV, in 1993. He became Associate Professor in 1999, and Full Professor in 2010, carrying out his teaching activities at the Telecommunications Engineering Faculty. $\mathrm{He}$ is with the Photonics Research Labs. (PRL), he is coauthor of more than 190 papers in journals and international conferences in the fields of optical delay line filters, Fiber Bragg Gratings, Microwave Photonics, Wavelength-DivisionMultiplexing (WDM) and Optical Code Division Multiple Access (OCDMA) devices and techniques. His current technical interests include complex Fiber Bragg Grating (FBG) design for signal processing, fiber sensors and OCDMA, and Photonic Integrated Circuits (PICs) applied to telecommunication and sensing.

Margarita Varón was born in Cartagena, Colombia. She received the B.Eng. degree in electronic engineering from Universidad Javeriana, Bogotá, Colombia, in 2000, the M.Sc. degree in optical communications and photonic technologies from Politecnico di Torino, Turin, Italy, in 2003, and the Ph.D. degree in optoelectronics from ISAE-SUPAERO, Toulouse, France, in 2008. Since 2010, she has been with Universidad Nacional de Colombia, in Bogotá Colombia. Her main areas of research interest are optoelectronics, particularly optical fiber sensors and optoelectronic oscillators. 